\title{
Training in liaison psychiatry
}

\section{Recommendations from the Liaison Psychiatry Group Executive Committee}

It is generally agreed that training in liaison psychiatry should be available at $\mathrm{SHO} /$ registrar grade, but the College's guidelines are not specific about what it might entail. As a result it is difficult for approval visitors to decide whether a particular post on a rotational training scheme truly provides liaison experience. At times so-called "liaison experience" amounts to no more than participation in a duty-rota for visiting a general hospital to undertake the assessment of cases of deliberate self-harm.

Senior registrars need guidance on what constitutes adequate training for a post at consultant level with an interest in [and sessional commitment to] liaison work. By the same token, appointments advisory committees need to know what criteria to apply when assessing the training of a candidate who is applying for such a post.

The executive committee of the liaison psychiatry group believes that the College's guidelines on training at SHO/register grade, and the JCHPT's guidelines on training at senior registrar grade, should be more specific than they are. The group has agreed criteria for training in liaison psychiatry, and they are outlined in this paper. We are aware that many schemes do not (and could not in the short-term) offer placements which met these criteria. Nevertheless, it is our view that they are the minimum which need to be satisfied before a post is accepted as providing liaison psychiatry training.

\section{Training at $\mathrm{SHO} /$ registrar grade Identified liaison psycó.iatry placements}

Every training scheme should offer at least one six month placement during which the trainee obtains experience in liaison psychiatry work with adults [16-65 years]. Similar placements may also be available as part of training in old-age psychiatry but cannot substitute for experience with younger adults. It would be desirable for the liaison experience to be provided during a full-time specialist placement; a minimum of four sessions per week liaison experience during a placement with mixed responsibilities would be acceptable. Clinical liaison experience requires contact with the general hospital as part of the trainees daily activities, and not just visiting the hospital ad hoc.

\section{Defined liaison psychiatry experience}

The clinical experience obtained should include work in all of the following areas:

(a) assessment of cases of deliberate self-harm

(b) assessment and management of patients referred from [and seen in] a medical setting, with:

(1) co-existent psychopathology and physical illness

(2) problems of adjustment to chronic illness

(3) unexplained physical symptoms and other forms of somatisation

(4) disorders of the nervous system or other disorders leading to transient or permanent cerebral dysfunction

(c) assessment of acute psychiatric cases, use of the Mental Health Act, and crisis management in the $A+E$ department and elsewhere in the general hospital.

\section{Identified clinical supervision}

The trainee's clinical work in liaison psychiatry should be undertaken with adequate supervision, which has the following characteristics.

(a) It is undertaken by a designated supervisor (not simply the duty psychiatrist of the day) who is a consultant with a special commitment to liaison psychiatry.

(b) There is a regularly timetabled face-to-face supervision session with the designated consultant. In addition there should be supervision of cases at the time they are seen, which must be readily available on request.

(c) There is a component of clinical teaching in the timetabled supervision. Such teaching should include theoretical and practical aspects of psychosomatic medicine and liaison psychiatry, relevant to the cases seen in the general hospital.

\section{Training at senior registrar grade}

Ideally the trainee should have had clinical experience in liaison psychiatry at $\mathrm{SHO} /$ registrar level of the sort outlined above. Where such experience has not been obtained at SHO/registrar grade, it should be obtained at senior registrar level under appropriate supervision. 
In addition, the following types of experience should be included in a senior registrar training placement.

Clinical

(a) Supervision by a named individual consultant with a special responsibility for providing the liaison service.

(b) Close liaison links should be established with at least one clinical department during the attachment.

(c) Out-patient work should be undertaken in a specialist liaison clinic held on the general hospital site.

(d) The opportunity should be available to supervise other professionals who are undertaking liaison work.

Education (a) Participation in and attendance at case presentations and other joint teaching meetings held in other clinical departments. (b) Experience in teaching medical staff such as house physicians and $A+E$ staff.

(c) It is desirable for the SR to have the opportunity to undertake supervised research in an area of liaison psychiatry.

Management (a) Experience of coordinating the running of at least one part of the liaison service.

(b) Experience in developing and completing at least one audit project.

The exact distribution of clinical sessions will depend on the local contract, but should amount to the equivalent of six sessions per week during a placement of at least 12 months.

A. O. House

Secretary of the

Liaison Psychiatry Group

F. CReEd

Chairman of the

Liaison Psychiatry Group

\section{Trainees' forum}

\section{European Trainees Conference}

\section{Michael van Beinum, European Working Party of the Collegiate Trainees Committee}

Trainees in psychiatry from across Europe met to explore psychiatric training issues at a conference organised by the Collegiate Trainees Committee of the Royal College of Psychiatrists on 29 June 1992 in London. Altogether 16 delegates came, representing nine different countries. Each delegate had been put forward by their own country's training organisation and was able to represent the view of the trainees in that country. There were several aims in having this meeting: to provide a forum in which to learn about the diversity and richness of the current training of psychiatrists in Europe; to explore ways in which trainee psychiatrists can promote and improve their own training in a European context; and to develop organisational structures that would give psychiatric trainees a voice in the evolution of European training standards.
There was a great diversity of training in Europe, not only for postgraduate doctors specialising in psychiatry, but also at medical undergraduate level. Both the boundaries of psychiatry and how best to train in the speciality varied greatly between countries. Child and adolescent psychiatry was a separate training in several countries, e.g. Finland and Germany, but part of general psychiatric training in the UK. Neurology was seen as an essential part of training in psychiatry in a number of European countries, whereas in the UK neurology was seen as quite distinct from psychiatry and there was no expectation that a psychiatrist had such training. Psychotherapy was seen as one of the core skills of a psychiatrist by all delegates, and great surprise was expressed that psychotherapy training was not mandatory in the UK, the only country where this 